\title{
An unusual cause of an 'acute abdomen'
}

\author{
S C Satchell, T Riordan, M Beaman
}

A 16-year-old girl presented to her general practitioner after 4 days of a severe sore throat, difficulty in swallowing, muscle and joint aches and anorexia. There was no history of foreign travel or recent infectious disease contacts. She was found to have swollen cervical lymph nodes with a pyrexia and as a viral illness was diagnosed, no antibiotics were given. As the initial symptoms improved, she developed diarrhoea and abdominal pain which became severe, leading to hospital admission on the ninth day of illness. She was pyrexial at $38^{\circ} \mathrm{C}$ with a mildly infected pharyngeal mucosa, dehydration, a heart rate of 120 beats $/ \mathrm{min}$ and a blood pressure of $100 / 60 \mathrm{mmHg}$. There was generalised severe tenderness of the abdomen with guarding. She was disorientated but there were no localising neurological signs and no evidence of meningitis.

Investigations were as follows: haemoglobin $14.5 \mathrm{~g} / \mathrm{dl}$, platelets $50 \times 10^{9} / 1$, white blood cells 6.0 $\times 10^{9} / 1$, urea $18.1 \mathrm{mmol} / 1$, creatinine $182 \mu \mathrm{mol} / \mathrm{l}$, bilirubin $43 \mu \mathrm{mol} / 1$, albumin $26 \mathrm{~g} / \mathrm{l}$, international normalised ratio 2.5 , activated partial thromboplastin ratio 1.93 , erythrocyte sedimentation rate $42 \mathrm{~mm} / \mathrm{h}, \mathrm{C}$-reactive protein $422 \mathrm{mg} / \mathrm{l}$. A monospot test was negative. Urinalysis demonstrated blood and protein but microscopy and culture were negative. Plain abdominal and chest X-rays were normal apart from a small left pleural effusion. An abdominal ultrasound scan showed multiple fluid-filled loops of small bowel but no free fluid collections.

She became increasingly short of breath and on the following day required transfer to the intensive care unit where a further chest X-ray was performed (figure).

Department of Medicine, Royal Devon and Exeter Hospital, Exeter, UK

S C Satchell

M Beaman

Public Health

Laboratory, Church Lane, Heavitree,

Exeter, UK

T Riordan

\section{Correspondence to}

Dr S C Satchell, Renal Unit, Royal Devon and Exeter Hospital, Exeter EX2 5DW, UK

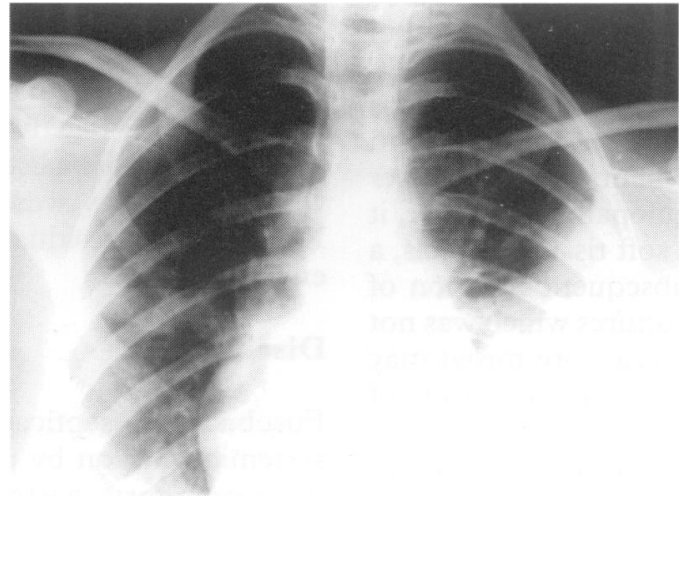

\section{Questions}

1 What was the differential diagnosis after the first day of admission?

2 What does the chest X-ray show?

3 How does this help in reaching the correct diagnosis?

Figure Antero-posterior erect chest X-ray 


\section{Answers}

\section{QUESTION 1}

At presentation there was a systemic inflammatory condition which included impairment of renal, hepatic and respiratory function with a coagulopathy. Although an acute vasculitis may rarely present in this way, the history was more suggestive of a microbial aetiology and the following diagnoses were considered:

Intra-abdominal sepsis with Gram-negative septicaemia was suggested by the striking abdominal component of the illness presentation. However, the ultrasound scan showed no specific abnormality such as evidence of perforation and this diagnosis would not account for the preceding upper airway symptomatology. Infection with the organisms usually responsible for gastro-enteritis such as salmonella, may be complicated by an acutely tender abdomen caused by colitis, and a typhoid-like septicaemic illness.

Staphylococcal toxin-induced illness was considered although the patient was not shocked according to the usual definition (systolic blood pressure $<90 \mathrm{mmHg}$ ). Prior sore throat has been reported in this condition and relative youth, fever, abdominal pain with diarrhoea, abnormal liver function tests and thrombocytopenia are common. The absence of a rash or obvious focus of infection such as tampon use $(92 \%$ of cases of toxic shock syndrome occur during menstruation) or a skin wound also argued against the diagnosis.

Streptococcal 'toxic shock' syndrome has rarely been reported to follow a tonsillitis and could cause renal impairment, abnormal liver function tests and a coagulopathy. However, it is usually associated with soft tissue necrosis, a rash, hypotension and subsequent isolation of streptococci from blood cultures which was not the case here. A streptococcal sore throat may directly precede peritonitis as a result of bacteraemic spread.

Infectious mononucleosis would explain the initial symptoms well and may be associated with severe abdominal pain due to splenic enlargement and rupture. Factors against this diagnosis include the negative monospot test, high C-reactive protein, coagulopathy and absence of atypical mononuclear cells. Splenic rupture and bleeding may be suggested in some cases by anaemia and free intraabdominal fluid detectable on ultrasound examination.

Fusobacterial septicaemia commonly affects multiple organs in a young, previously fit individual and classically occurs following an upper respiratory tract infection.

\section{QUESTION 2}

The chest X-ray shows bilateral pleural effusions and multiple rounded lung opacities consistent with abscess formation.

\section{QUESTION 3}

Multiple lung abscesses are commonly seen following aspiration occurring, for example, secondary to seizures or alcohol intoxication and are also associated with severe peri-odontal disease. Certain pneumonias may develop frank abscesses but in the absence of a preceding pneumonia or these other factors, haematogenous infection is the most likely explanation.

Staphylococcus aureus is the commonest cause of metastatic lung abscesses which have been reported with staphylococcal septicaemia in various situations such as right-sided endocarditis secondary to intravenous drug abuse and in septic thrombophlebitis. Abscesses are not a feature of staphylococcal toxic shock syndrome as this is not associated with bacteraemia. Streptococcal septicaemia only very rarely causes lung abscesses and although these have been documented secondary to intraabdominal sepsis they would normally be a very late manifestation.

The radiological features are, however, typical of fusobacterial septicaemia and bilateral intercostal drains produced foul-smelling pus containing Gram-negative rods. Although culture was negative, probably due to prior antibiotic administration, blood cultures taken on admission grew Fusobacterium necrophorum after $48 \mathrm{~h}$. Anaerobic streptococci were also subsequently isolated from the blood cultures.

Treatment was initiated with intravenous imipenem and clindamycin, and metronidazole was added on the second day. The patient was discharged from intensive care after 7 days but the swinging pyrexia up to $39^{\circ} \mathrm{C}$ persisted for 2 weeks. When discharged from hospital 1 month later on oral amoxycillin and metronidazole, the C-reactive protein was $104 \mathrm{mg} / 1$ and did not normalise for a further 2 months when antibiotics were discontinued. A chest $\mathrm{X}$-ray showed residual left basal pleural thickening.

\section{Discussion}

Fusobacterial septicaemias are the result of systemic invasion by commensal organisms of the respiratory, gastro-intestinal and genitourinary tracts. The most frequently described of these is that due to $F$ necrophorum, sometimes referred to as necrobacillosis. ${ }^{1}$ This case is characteristic of a sub-group of these first described in detail by Lemierre as occurring following an attack of acute tonsillitis. ${ }^{2}$ The history typically begins with a fever and a sore throat which may be mild and mistaken for a viral infection. In some cases there has been serological evidence of preceding or concurrent Epstein-Barr virus infection. ${ }^{3}$ Fusobacteria cross the inflamed mucosa gaining access to the tonsillar veins where thrombophlebitis may extend into the internal jugular vein. This may be subclinical or may have resolved by the time metastatic spread is manifest and is not essential for diagnosis. Because of the variety of possible sites to which the organism spreads, there is a wide range of clinical presentations. ${ }^{4}$ This potentially lifethreatening infection carried a mortality of up to $90 \%$ in the pre-antibiotic era ${ }^{2}$ and it is therefore important to be aware of the condition even though it is now rarely seen. 
The lungs are most commonly involved after systemic dissemination, resulting in chest pain and dyspnoea associated with multiple pulmonary septic infarcts, pleural effusions or empyema. Articular lesions are also frequent, such that Lemierre declared that involvement of these two systems following a sore throat with pyrexia and rigors to "constitute a syndrome so characteristic that mistake is almost impossible". ${ }^{2}$ However other presentations frequently do cause diagnostic confusion ${ }^{5}$ : these include thyroiditis, psoas abscess, meningitis, endocarditis, osteitis and renal impairment. ${ }^{1}$ Even the classical presentation is so rarely seen that a diagnosis of another infection is likely to be considered first. Suppurative peritonitis and abscess formation in the liver and spleen have been described ${ }^{2}$ but abdominal pain as the predominant symptom is rarely reported.

The diagnosis of Lemierre's syndrome is primarily clinical and should always be considered in a previously healthy young adult having a severe septicaemic illness following a sore throat. Resolution of upper respiratory tract symptoms should not discourage the diagnosis and a careful examination of the neck is mandatory as is a chest X-ray. Radiological imaging may confirm a suspicion of thrombophlebitis.

In vitro fusobacteria are sensitive to many antibiotics with the particular exception of aminoglycosides but clinical experience has shown that use of metronidazole leads to more rapid response. ${ }^{5}$ Penicillin is usually included in case of concurrent anaerobic streptococci infection which is seen not infrequently. ${ }^{2}$ Clindamycin was continued here as there is evidence that it is particularly effective in the treatment of anaerobic lung abscesses. Where possible abscesses should be drained and the ligation of a thrombophlebitic internal jugular vein in severe cases has been advocated. Generally, clinical resolution is slow, with the

1 Alston MD. Necrobacillosis in Great Britain. BMf 1955;1:1524-8.

2 Lemierre A. On certain septicaemias due to anaerobic organisms. Lancet 1936;1:701-3.

3 Moller K, Dreijer B. Post-anginal sepsis (Lemierre's disease): a persistent challenge. Presentation of 4 cases. disease): a persistent challenge.
Scand $\Im$ Infect Dis 1997;29:191-4.

4 Eykyn SJ. Necrobacillosis. Scand F Infect Dis 1989; 62(suppl): 41-6.

\section{Learning points}

- fusobacterial septicaemia is a rare but potentially life-threatening condition and awareness of the range of presentations is important to avoid

diagnostic delay; it should be considered particularly in a young adult with septicaemia and a recent sore throat

- examination of the neck and a chest X-ray are mandatory in patients with this history

- antibiotic treatment should be started expectantly as the organisms are slow to grow in culture and clinical response may be delayed; abscesses should be sought and drained

temperature and inflammatory markers taking over two weeks to normalise and the chest $\mathrm{X}$-ray may be abnormal for several months. With correct antibiotic treatment, mortality rates are now reported in the region of $5 \%{ }^{6}$ and full recovery may generally be expected. It is likely that early administration of antibiotics has been responsible for the reduction in recognition of the infection and in the incidence of the full-blown syndrome. ${ }^{7}$ This case demonstrates that while the organism is exquisitely sensitive to antibiotics, and therefore cultures taken after treatment has commenced are often negative, without early antibiotics a life-threatening septic illness with multiple organ impairment can readily develop.

\section{Final diagnosis}

Fusobacterium necrophorum septicaemia with metastatic manifestations following tonsillitis (Lemierre's syndrome).

Keywords: Fusobacterium necrophorum; necrobacillosis; Lemierre's syndrome

\footnotetext{
5 Moore-Gillon J, Lee TH, Eykyn SJ, Phillips I. Necrobacillosis: a forgotten disease. BMf 1984;288:1526-7. 6 Sinave CP, Hardy GJ, Fardy PW. The Lemierre syndrome: suppurative thrombophlebitis of the internal jugular vein secondary to oropharyngeal infection. Medicine 1989;68: 85-94.

7 MacDonald AA, Harar RP, Prior AJ. Necrobacillosis: are we missing the early stages of this life-threatening infection? (letter). Lancet 1995;346:1705.
} 